\title{
Article
}

\section{Investigation on the Effect of Femtosecond Laser Induced Spin Transfer Torque of GdFeCo Alloy}

\author{
Haozhe Huang ${ }^{1}{ }^{\oplus}$, Haiwei Wang ${ }^{1, *}{ }^{\mathbb{C}}$, Zhihao Zeng ${ }^{1}$, Rongyao Wang ${ }^{1}$, Xinyu Zhang ${ }^{2}$, Weiming Cheng ${ }^{3}$ and \\ Changsheng Xie ${ }^{1}$ \\ 1 Wuhan National Laboratory of Optoelectronics, Huazhong University of Science and Technology, \\ Wuhan 430074, China; hhz_ozn@hust.edu.cn (H.H.); mizhengzzh@hust.edu.cn (Z.Z.); \\ m201972975@hust.edu.cn (R.W.); cs_xie@hust.edu.cn (C.X.) \\ 2 School of Automation, Huazhong University of Science and Technology, Wuhan 430074, China; \\ x_yzhang@hust.edu.cn \\ 3 School of Optical and Electronic Information, Huazhong University of Science and Technology, \\ Wuhan 430074, China; wmcheng@hust.edu.cn \\ * Correspondence: hiway@hust.edu.cn
}

check for updates

Citation: Huang, H.; Wang, H.; Zeng, Z.; Wang, R.; Zhang, X.; Cheng, W.; Xie, C. Investigation on the Effect of Femtosecond Laser Induced Spin Transfer Torque of GdFeCo Alloy. Appl. Sci. 2021, 11, 6501. https:// doi.org/10.3390/app11146501

Academic Editor: Yurii K. Gun'ko

Received: 31 May 2021

Accepted: 8 July 2021

Published: 15 July 2021

Publisher's Note: MDPI stays neutral with regard to jurisdictional claims in published maps and institutional affiliations.

Copyright: (c) 2021 by the authors. Licensee MDPI, Basel, Switzerland. This article is an open access article distributed under the terms and conditions of the Creative Commons Attribution (CC BY) license (https:// creativecommons.org/licenses/by/ $4.0 /)$.
Featured Application: All-Optical magnetization recording technology.

Abstract: All-optical magnetic switching (AOS) provides a novel approach to improve writing ability and energy efficiency compared to those utilized in the mainstream magnetic data storage products. Rare earth-transition metals (RE-TM) exhibit extremely fast magnetization switching induced by one single incident linearly polarized laser pulse; however, the mechanism is still ambiguous. Here, we show by atomistic spin simulation that the laser induced spin transfer torque dominates the magnetization reversal of Fe sublattice in $\mathrm{Gd}_{25} \mathrm{Fe}_{75}$ alloy, and that the switching speed of $\mathrm{Gd}_{25} \mathrm{Fe}_{75}$ alloy is relevant to the amount of spin current. This implies that a possible helicity independent mechanism underlies the RE-TM alloy AOS process. We also find that the greater the spin current density the faster the magnetization switching, and the time magnetization reversal of $\mathrm{Gd}$ and Fe takes is also affected by the spin current density.

Keywords: GdFeCo; alloy; atomistic spin simulation; spin transfer torque

\section{Introduction}

The exploding amount of data is devouring the capacity of storage devices and demanding ever faster read/write speed of these devices. For the time being, most of the data generated is stored on hard disk drives. However, the recording density of these drives is approaching the limit of the magnetic material due to the superparamagnetic effect [1]. Thus, turning to materials with higher coercivity is necessary for greater recording density. On the other hand, the magnetic field for writing is limited by the size of write head, which is in turn limited by the expected recording density. The writing speed of hard disk drives is also limited by the speed of magnetization switching. Therefore, it has become a significant challenge to develop new approaches suitable for scenarios featured with high coercivity media and ultrafast write speed. All-optical magnetic switching (AOS), which utilizes ultrafast femtosecond laser pulses to excite magnetic recording materials rather than traditional magnetic field, has drawn much attention from researchers [2-7]. For one thing, data is written by one or a series of laser pulses, so it is possible to exploit some unique traits of the optical field in space; hopefully this will overcome the coercivity of the recording materials. Another reason is that theoretically the size of laser spots can be shrunk by means of surface plasmon polaritons (SPPs) [8,9], so that the recording spot size beyond diffraction limit can be achieved, thus greatly improving the storage density of disks [10,11]. Experiments also show that magnetization can be reversed by a single 
femtosecond laser pulse [2] repeatedly, which means the write/overwrite convenience of traditional magnetic recording is retained by AOS. According to the helicity of the excited laser pulse, AOS can be divided into two categories: helicity dependently AOS (HD-AOS) and helicity independently AOS (HI-AOS).

Laser intruded magnetization switching has been observed in many rare earthtransition metals (RE-TM), including $\mathrm{Tb} / \mathrm{Co}$ multilayers, $\mathrm{TbCo}$ alloy, $\mathrm{TbFe}$ alloy and GdFeCo alloy [12]. Among them all, GdFeCo is the one explored most both in HD-AOS and HI-AOS. There is no lack of studies on the internal mechanism of GdFeCo material AOS to explain its spin switching dynamic process [13-17], but most of these studies concluded that the magnetization switching was related to the helicity of femtosecond laser pulse because an axial magnetic field can be generated by intense circularly polarized light through what is known as the inverse Faraday effect [18-20]. Stanciu et al. found that a single $40 \mathrm{fs}$ circularly polarized laser pulse can cause GdFeCo alloy film magnetization reversal for the first time, the different phase of spin precession would be excited by circularly polarized lasers in different directions of helicity [2]. They deemed that AOS is not completed by absorbing the angular momentum of photons and there is another ultrafast non-thermal coupling between spin and photon angular momentum in GdFeCo alloy. On the other hand, some researchers discovered that AOS can be stimulated by linearly polarized single laser pulse in metallic ferrimagnet $\mathrm{GdFeCo}$. The process of HI-AOS can be divided into two consecutive steps: sublattices demagnetization and sublattices spin reversal. Although there is a plethora of research devoted to the demagnetization, and many breakthroughs have been made, the physical mechanism and explanation of spin dynamics in spin reversal are still ambiguous. The thermal induced magnetization switching (TIMS) theory proposed by Ostler et al. can explain why the spins of different sublattices demagnetize successively, but are not able to provide a reasonable explanation for the stable switching of Fe spins. In TIMS theory, the thermal effective field is stochastic, and the thermal fluctuations on each atomic site can be described by a Gaussian white noise term. In the X-ray magnetic circular dichroism (XMCD) experiment observed by Ostler et al., the sublattice spins of Fe and the sublattice spins of $\mathrm{Gd}$ showed a transient ferromagnetic-like state and this means that the spins of the two different elements remain magnetized in the same direction. The occurrence of this state needed to overcome the exchange interaction between Fe and Gd, but the thermal perturbation has no direct impact on the magnetization of Fe. Therefore, the mechanism of HI-AOS in ferromagnetic materials with different sublattices remains to be studied further.

Variations in the flow of spin-angular momentum occur when spin-polarized electrons pass through a magnetic domain wall or any other spatially non-uniform magnetization distribution. In this process, the spins of the charge carriers rotate to follow the local magnetization, so the spin vector of the angular momentum flow changes as a function of position. The magnetization of the magnetic medium changes the flow of spin angular momentum by exerting a torque on the flowing spins to reorient them, and therefore the flowing electrons must exert an equal and opposite torque on the magnetic medium. This torque applied by non-equilibrium conductive electrons onto a magnetic medium is called spin transfer torque (STT) [21-24]. Ultrafast diffraction experiments using X-ray were performed by Graves et al. to investigate the nanoscale spin dynamics of GdFeCo amorphous alloy stimulated by femtosecond lasers $[25,26]$. In this nanoscale chemical and magnetic inhomogeneous alloy, the experiment results show in the first picosecond, and the angular momentum is transferred from the larger Fe-rich region to the smaller Gd-rich region, resulting in the spin reversal of the Gd. They deemed that this local transfer of angular momentum might be caused by spin current. Spin-polarized current is often measured by magnetic tunnel junction (MTJ) structure in experiments, and thus the researchers are usually focused on the multi-layer structure materials containing the self-selecting valve, but the spin current effect in alloy materials is rarely studied. In fact, the physical mechanism of spin transfer torque varies. Researchers have shown that the 
optical spin transfer torque $[27,28]$, spin-dependent Seebeck effect [29-31] and laser pulse excite superdiffusive spin current $[32,33]$ can all cause the spin transfer torque effect.

In order to better clarify the ultrafast laser single pulse induced spin transfer torque effect in GdFeCo amorphous films, we take both ultrafast thermal effect and spin transfer torque effect into account and calculate the magnetization dynamics for $\mathrm{Gd}_{25} \mathrm{Fe}_{75}$ amorphous alloy films. The results show that the spin transfer torque may be the critical factor that causes reversal of spin magnetization of Fe sublattice prior to Gd sublattice during helicity, independent of photomagnetization of GdFeCo amorphous alloy films.

\section{Method}

A Co-Cu-Co three-layer structure model was constructed by Aquino et al. [34] to describe the macro spin transfer torque effect. The term corresponding to the effect of spin transfer torque was added to the LLG equation to study the magnetization dynamics' influence of the spin current on the magnetic free layer in the model [35]. A three-layer structure is schematically shown in Figure 1 . The first layer is a magnetically fixed layer with a magnetization intensity of $p$ and a fixed direction. The second one is a film composed of nonmagnetic conductor; the third one is a magnetically free layer, with magnetization intensity $m$ and freely altering direction. After the unpolarized current enters the fixed layer, the electron spin is polarized, forming a spin-polarized current with the same direction as $p$. The spin-polarized current from the fixed layer flows into the free layer, and then the spin-polarized electrons interact with the free layer media, causing the magnetization direction and magnitude of the free layer to change.

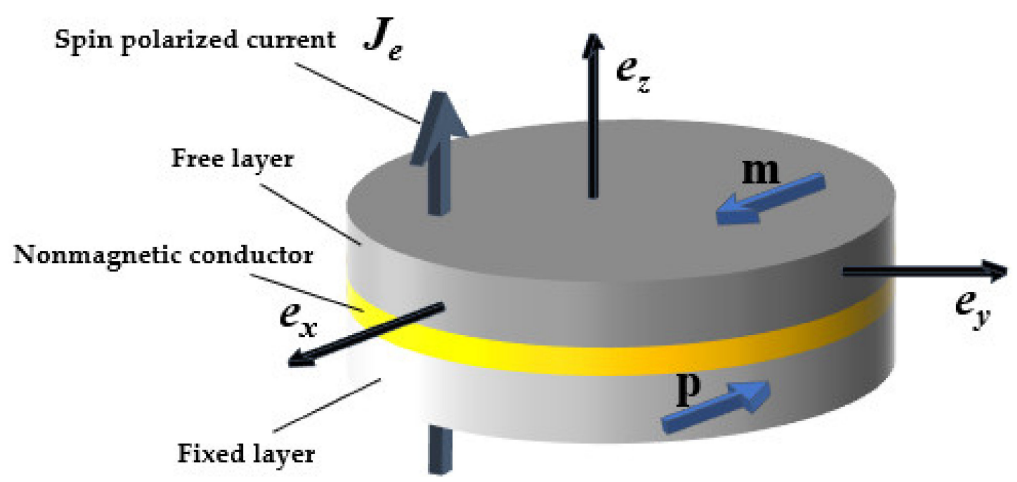

Figure 1. Schematic diagram of three-layer structure model.

The exploration of magnetization dynamics of ferromagnetic materials is mainly to study the interaction between different sublattice materials. Since Co element in GdFeCo alloy films mainly plays the role of adjusting Curie temperature and compensating temperature, it has little effect on other properties of materials, but it will greatly increase the complexity of the model and the amount of calculation. Therefore, in this study, Co and Fe were regarded as the same sublattice, which is a transition metal element. In order to reduce the computational complexity, the object of numerical simulation is $\mathrm{Gd}_{25} \mathrm{Fe}_{75}$ alloy and it was assumed to be amorphous, in which the spins of Gd and Fe are randomly distributed in the periodic structure, The GdFe alloy atomic model also can reflect both the nature of magnetic interaction and the ferromagnetic properties of the material.

In the atomistic model of GdFe alloy, rare earth elements and transition metal elements can be considered as the fixed layers and free layers in the three-layer structure respectively, while the non-magnetic conductor in the middle of the three-layer structure only plays the role of transporting the spin current. Therefore, it is not necessary to consider the role of the interlayer when considering the sublattice magnetization kinetics of GdFe alloys.

In our study, the direction of spin polarization is related to the initial magnetization direction of the Gd sublattice magnetization. The whole process can be described in Figure 2. 


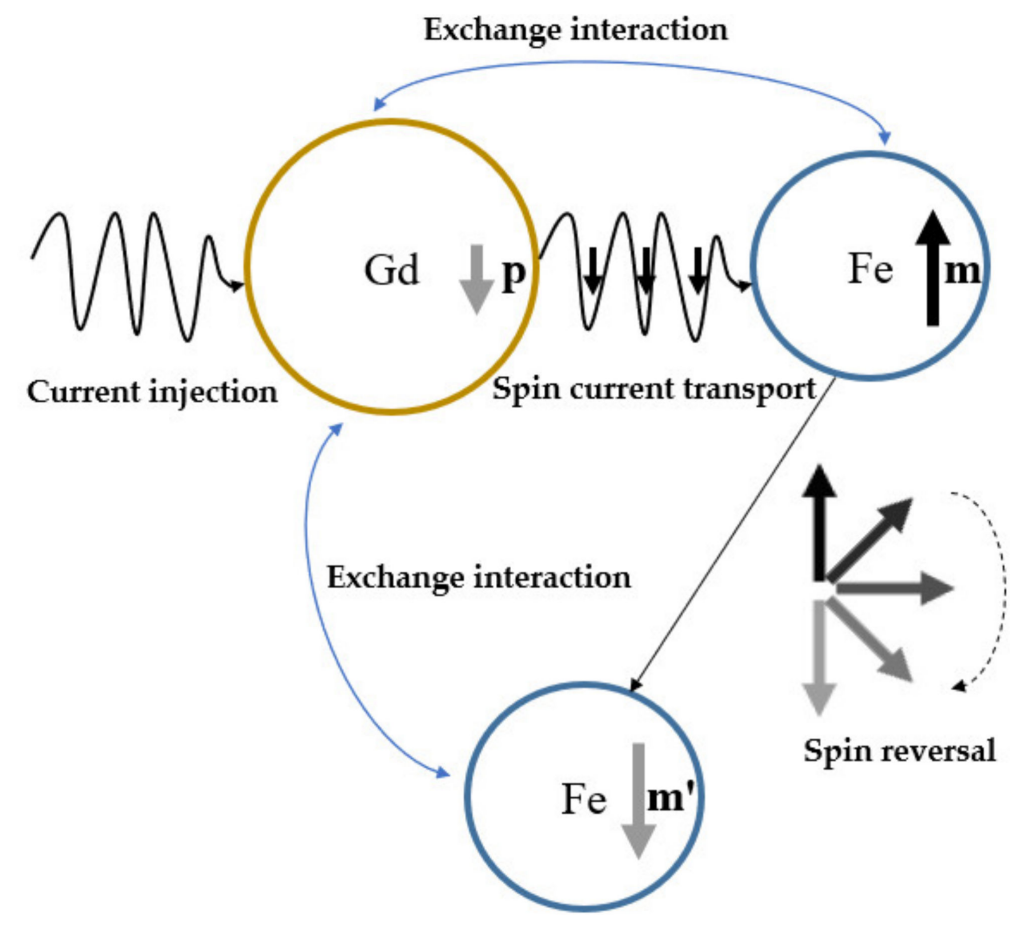

Figure 2. Spin current conduction in a GdFe alloy.

Ultrafast linearly polarized femtosecond laser acts on GdFeCo amorphous alloy thin films. Due to the temperature gradient effect, a certain order of magnitude of excited electrons are generated. The injection of electrons into the $\mathrm{Gd}$ sublattice results in a spin-polarized current. The spin transfer torque effect induced by spin-polarized current impacts on the spin of the Fe sublattice results in the spin reversal of the Fe sublattice first. The electron temperature then drops rapidly below Curie temperature, and the exchange interaction between the Fe and Gd causes the spin reversal of the Gd sublattice. To this end, a mathematical model describing the magnetic physical process is established.

Our model for GdFe amorphous alloy, based on the extended Heisenberg spin Hamiltonian. included the uniaxial single-ion anisotropy energy and the exchange interaction energy:

$$
\mathcal{H}=\mathcal{H}_{a n i}+\mathcal{H}_{\text {exc }}=-k_{u} \sum_{i}\left(\boldsymbol{S}_{i} \cdot \boldsymbol{e}\right)^{2}-\sum_{i<j}^{N} J_{i j} S_{i} \cdot S_{j}
$$

where $\mathcal{H}_{a n i}$ is the spin Hamiltonian for uniaxial anisotropy energy, $\mathcal{H}_{\text {exc }}$ is the Hamiltonian for exchange interaction energy, $k_{u}$ is the anisotropy constant, $S_{i}$ is a unit vector representing the direction of atomic spin moment, $S_{j}$ represents the other atoms besides $S_{i}$ in the range of exchange interactions, $e$ is a unit vector describing the direction of the easy axis, $J_{i j}$ is the exchange interaction between atomic sites $i$ and $j$.

For all the calculations in this paper, the initial magnetization direction of GdFe alloy is along the $\mathrm{Z}$ axis in Cartesian coordinates. In order to balance the two conditions of lower Curie temperature and higher magnetic moment, the ratio of transition metal elements to rare earth elements is generally 3:1 in the practical application of magneto-optical recording. We built the atomic models according to this elements ratio. Figure 3 shows the atomic-scale model structures of different sizes, with side lengths of $1 \mathrm{~nm}$ and $10 \mathrm{~nm}$ respectively, which were established according to the Hamiltonian of GdFe amorphous alloy thin films system in Equation (1). The value and unit of critical physical parameters in atomistic models of different sizes are exactly the same, as shown in Table 1. 


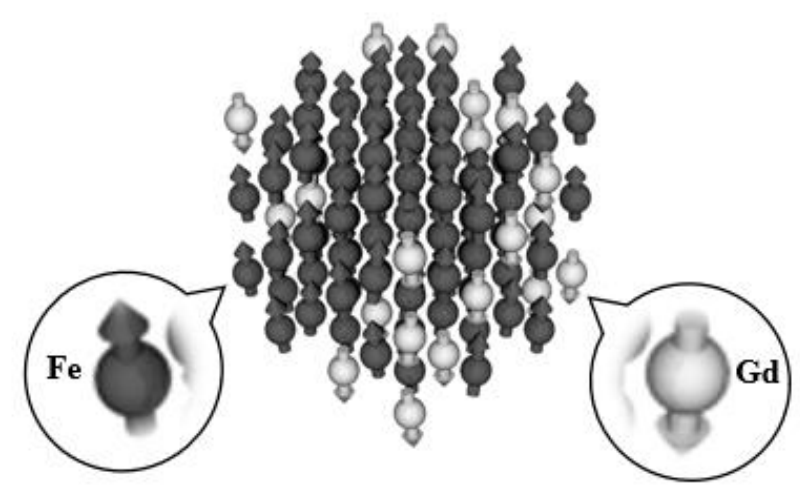

(a) $1 \times 1 \times 1 \mathrm{~nm}^{3}$

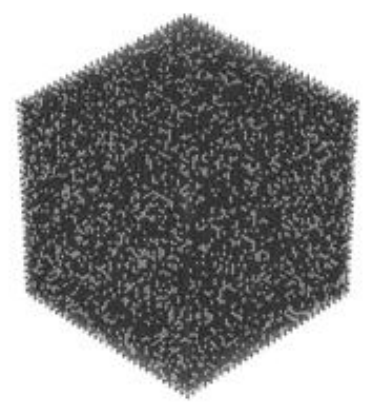

(b) $10 \times 10 \times 10 \mathrm{~nm}^{3}$

Figure 3. Atomistic models structure of $\mathrm{Gd}_{25} \mathrm{Fe}_{75}$ amorphous alloys with different sizes. (a) $\mathrm{Gd}_{25} \mathrm{Fe}_{75}$ amorphous cubic alloy with side length of $1 \mathrm{~nm}$, in which the spin of Fe atom is black and the spin of $\mathrm{Gd}$ atom is gray; (b) $\mathrm{Gd}_{25} \mathrm{Fe}_{75}$ amorphous cubic alloy with side length of $10 \mathrm{~nm}$.

Table 1. The values and units of critical physical parameters in atomistic models of different sizes.

\begin{tabular}{cccc}
\hline Parameter & Symbol & Value & Unit \\
\hline exchange constant of Gd-Fe & $J_{G d-F e}$ & $-1.09 \times 10^{-21}$ & $\mathrm{~J} /$ link \\
exchange constant of Gd-Gd & $J_{G d}$ & $1.26 \times 10^{-21}$ & $\mathrm{~J} /$ link \\
exchange constant of Fe-Fe & $J_{F e}$ & $2.835 \times 10^{-21}$ & $\mathrm{~J} /$ link \\
anisotropy energy constant & $k_{u}$ & $8.072 \times 10^{-24}$ & $\mathrm{~J} /$ atom \\
spin moment of Gd & $\mu_{G d}$ & 7.63 & $\mu_{\mathrm{B}}$ \\
spin moment of Fe & $\mu_{F e}$ & 1.92 & $\mu_{\mathrm{B}}$ \\
\hline
\end{tabular}

The magnetization dynamics are based on atomic-scale Landau-Lifshitz-Gilbert (LLG) equation. The spin variation of Fe sublattice is described by Equation (2), and the spin change of Gd sublattice is described by Equation (3):

$$
\begin{gathered}
\frac{\partial S_{i}}{\partial t}=-\frac{\gamma}{\left(1+\lambda^{2}\right)}\left[\boldsymbol{S}_{i} \times \boldsymbol{H}_{e f f}^{i}{ }^{\prime}+\lambda S_{i} \times\left(\boldsymbol{S}_{i} \times \boldsymbol{H}_{e f f}^{i}{ }^{\prime}\right)+\frac{J_{e} b(\boldsymbol{p} \cdot \boldsymbol{m})}{\gamma e M_{s}} \boldsymbol{S}_{i} \times\left(\boldsymbol{S}_{i} \times \boldsymbol{S}_{j}\right)\right] \\
\frac{\partial \boldsymbol{S}_{i}}{\partial t}=-\frac{\gamma}{\left(1+\lambda^{2}\right)}\left[\boldsymbol{S}_{i} \times \boldsymbol{H}_{e f f}^{i}{ }^{\prime}+\lambda \boldsymbol{S}_{i} \times\left(\boldsymbol{s}_{i} \times \boldsymbol{H}_{e f f}^{i}{ }^{\prime}\right)\right]
\end{gathered}
$$

where $\lambda$ is the microscopic damping parameter, $\gamma$ is the gyromagnetic ratio, $J_{e}$ is the current density, $\boldsymbol{H}_{e f f}^{i}{ }^{\prime}=\zeta_{i}-\partial \mathcal{H}_{i} / \partial S_{i}$ is the effective field at site $i$ and includes stochastic thermal fluctuations, $e$ is the absolute value of the electron charge, $M_{S}$ is the saturation magnetization for $\mathrm{Fe}, \mid b(\boldsymbol{p} \cdot \boldsymbol{m})=\left[-4+(1+P)^{3}(3+\boldsymbol{p} \cdot \boldsymbol{m}) / 4 P^{3 / 2}\right]^{-1}$ is a scalar function associated with magnetization unit $p$ and $m, p$ and $m$ represent the initial spin magnetization directions of Gd and Fe r2espectively. For the third term to the right of Equation (2), we define it as the atomic spin transfer torque term $\tau$ described as Equation (4). Equation (4) is corrected according to the macro current-induced magnetization dynamics proposed by $\mathrm{d}^{\prime}$ Aquino et al. [23,34,36], the macro format is used to describe spin transfer torque of two different magnetic layers and we corrected it to the atomic format.

$$
\tau=\frac{J_{e} b(\boldsymbol{p} \cdot \boldsymbol{m})}{\gamma e M_{S}} S_{i} \times\left(S_{i} \times S_{j}\right)
$$

We used the two-temperature model to simulate the effect of femtosecond laser heating on the electron-lattice temperature of the sample and the thermal coupling between the electrons and the lattices. The volume of the simulation for $\mathrm{Gd}_{25} \mathrm{Fe}_{75}$ alloy is $10 \times 10 \times 10 \mathrm{~nm}^{3}$, 
and as the laser acts on the whole space, the spin magnetization will be influenced in the whole space scope. The width of laser pulse is $60 \mathrm{fs}$ and the wavelength is $800 \mathrm{~nm}$. The balance temperature is $300 \mathrm{~K}$ and the time step of integration is $1 \mathrm{fs}$. The expression of the two-temperature model is as follows:

$$
\begin{aligned}
C_{e}\left(T_{e}\right) \frac{\partial T_{e}}{\partial t}= & \nabla\left[k_{e}\left(T_{e}\right) \nabla T_{e}\right]-G\left(T_{e}-T_{l}\right)+S(r, t) \\
& C_{l}\left(T_{l}\right) \frac{\partial T_{l}}{\partial t}=G\left(T_{e}-T_{l}\right)
\end{aligned}
$$

where $C_{e}=\gamma_{e} T_{e}$ and $C_{l}$ are the heat capacities of electrons and lattices respectively; $\gamma_{e}$ is the electron heat capacity constant; $T_{e}$ and $T_{l}$ are the temperatures of electrons and lattices respectively; $G$ is the electron-lattice coupling factor; $k_{e}$ is the electron heat conduction coefficient; $S(r, t)$ is the incident power density of the femtosecond laser light source, and its value is related to the spot radius and the irradiation duration. When the two-temperature model is used to study the AOS, the temperature of the electron is generally taken as the benchmark because the electron has a smaller heat capacity, and the temperature rises faster after being excited by the laser, and the peak value is also higher. The physical parameters of the atomic-scale LLG equation and two-temperature model are given in Table 2. Due to the difference in heat capacity values, the change rate of electrons and lattices temperature are very different; the temperature of lattices will lag much behind the temperature of electrons. Thus, when the electronic temperature exceeds the Curie temperature of the material, the material begins to demagnetize, while the lattice temperature slowly rises to just above room temperature to ensure that the material's structure will not be destroyed by the ultrafast laser.

Table 2. The physical parameters in two-temperature model.

\begin{tabular}{cccc}
\hline Parameter & Symbol & Value & Unit \\
\hline gyromagnetic ratio & $\gamma$ & $1.76 \times 10^{11}$ & $\mathrm{~T}^{-1} \mathrm{~S}^{-1}$ \\
microscopic damping parameter & $\lambda$ & 0.01 & - \\
electron heat capacity constant & $\gamma_{e}$ & 222 & $\mathrm{~J} \mathrm{~m}^{-3} \mathrm{~K}^{-2}$ \\
electron heat capacity & $C_{e}$ & - & $\mathrm{J} \mathrm{m}^{-3} \mathrm{~K}^{-1}$ \\
phonon heat capacity & $C_{l}$ & $2.3 \times 10^{6}$ & $\mathrm{~J} \mathrm{~m}^{-3} \mathrm{~K}^{-1}$ \\
Electron-phonon coupling factor & $G$ & $6.6 \times 10^{17}$ & $\mathrm{~W} \mathrm{~m}^{-3} \mathrm{~K}^{-1}$ \\
\hline
\end{tabular}

In order to simulate the pumping generation and attenuation of spin current in the actual situation, we fit the equation of current attenuation with incident laser pumping based on the previous experimental results [25,26]. The equation is given as follows:

$$
J_{e}=A J_{0}\left(1-e^{-\frac{t}{\tau_{a}}}\right)^{3} e^{-\frac{t}{\tau_{b}}}
$$

where $J_{0}$ is the maximum injection current density; $A$ is the amplitude amplification factor; $\tau_{a}$ and $\tau_{b}$ are the current pumping constant and current attenuation constant respectively.

\section{Results and Discussions}

In Figure 4, the normalized magnetization of $\mathrm{Gd}_{25} \mathrm{Fe}_{75}$ alloy induced by different incident laser power densities and injection current densities are presented quantitatively. When the incident laser power density is higher than $20 \mathrm{~mJ} / \mathrm{cm}^{2}$, the magnetization is unstable; this may be that the laser power density is too high and the electronic temperature can't drop to below the Curie temperature in a short time. Therefore, we chose to study the case of laser power density of 10 and $20 \mathrm{~mJ} / \mathrm{cm}^{2}$ to further study the influence of spin transfer moment effect on AOS process. 
(a)

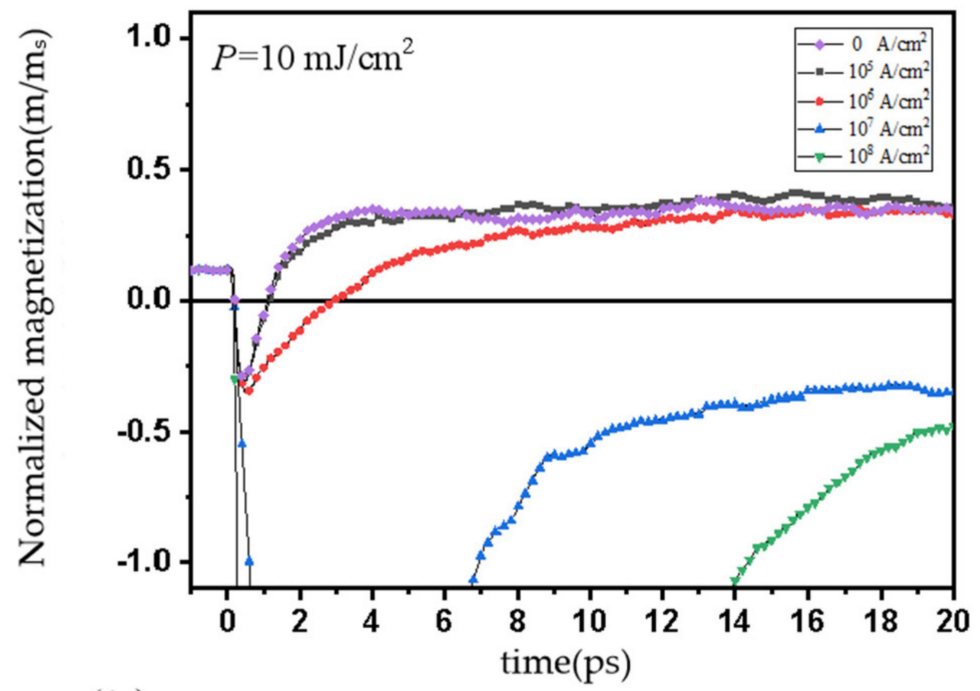

(b)

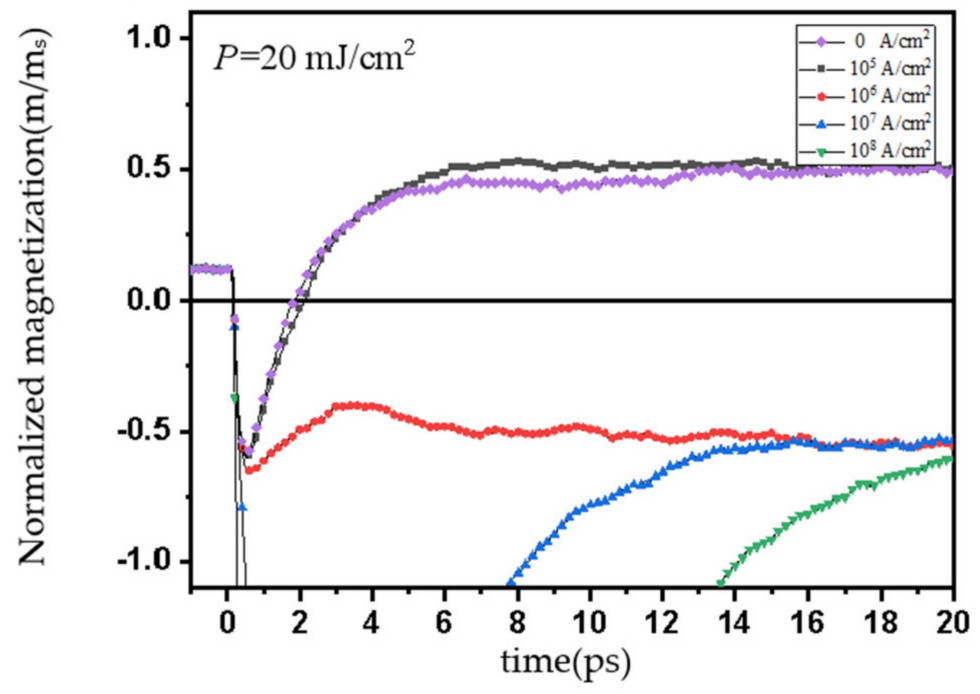

Figure 4. Normalized magnetization of $\mathrm{Gd}_{25} \mathrm{Fe}_{75}$ alloy induced by different incident laser power density and injection current density, the current density ranges from $0 \sim 10^{8} \mathrm{~A} / \mathrm{cm}^{2}$. (a) when the incident laser power density is $10 \mathrm{~mJ} / \mathrm{cm}^{2}$; (b) when the power density of the incident laser is $20 \mathrm{~mJ} / \mathrm{cm}^{2}$.

The current density of $0 \mathrm{~A} / \mathrm{cm}^{2}$ means that the spin transfer torque effect brought by the current is not considered at this time, instead, the thermal effect of the laser pulse on the material is considered. The magnetic system is in a stable state in the initial condition. When the laser pulse acts (at $0 \mathrm{ps}$ ), the electron temperature rises rapidly, and the magnetization of Fe and Gd sublattices are close to the demagnetization state, leading to a turning angle. Because of the laser pulse effect has a shot duration, the electron temperature will drop rapidly, and the magnetization of Fe and Gd sublattices will gradually return to the initial state. This requires special attention, and the magnetization of GdFe alloy will occur a short time reversal in 0 and 1 ps intervals (Figure $4 a, b$ ) because the atomic magnetic moment of $\mathrm{Gd}$ is $7.63 \mu_{\mathrm{B}}$, far greater than $1.92 \mu_{\mathrm{B}}$ of Fe; thus, under the initial state, the magnetization direction of the GdFe alloy system is the same as Gd sublattice. After demagnetization, the magnetization Gd and Fe are close to zero, but a higher proportion of Fe is in the alloy system. Therefore, for a short period of time, the magnetization of the GdFe alloy system will show the same direction as Fe sublattice. 
When the incident laser power is $10 \mathrm{~mJ} / \mathrm{cm}^{2}$, the injection current density of $10^{6} \mathrm{~A} / \mathrm{cm}^{2}$ does not make the magnetization of $\mathrm{Gd}_{25} \mathrm{Fe}_{75}$ alloy switch, but the injection current density of $10^{7} \mathrm{~A} / \mathrm{cm}^{2}$ can make the magnetization of $\mathrm{Gd}_{25} \mathrm{Fe}_{75}$ alloy switch, which means the threshold value of the flipped current density is between $10^{6}$ and $10^{7} \mathrm{~A} / \mathrm{cm}^{2}$. When the incident laser power is $20 \mathrm{~mJ} / \mathrm{cm}^{2}$, the injection current density of $10^{6} \mathrm{~A} / \mathrm{cm}^{2}$ can make the magnetization of $\mathrm{Gd}_{25} \mathrm{Fe}_{75}$ alloy reverse, and the stabilization time after the magnetization reversal becomes longer with the increase of the injection current density, and the magnetization intensity after stabilization is almost unaltered.

The results show that within a certain range of the laser power density, the higher the incident laser power density, the lower the injection current power density required for switching, and there may be an optimal injection current density window, making it possible for switching to occur and be able to achieve stability at the fastest speed; this is of great significance to the reading and writing of high speed all-optical magnetic recording devices in actual application. The simulated results are of the same order of magnitude as Iacocca et al. had estimated photoelectric current density in GdFeCo alloy, which further indicates that the light induced spin current in GdFeCo alloy may indeed exist in the process of photoinduced magnetic transformation.

To further understand the physical mechanism, it is necessary to study the magnetization dynamics of the two sublattices under the influence of spin transfer moment effect after analyzing the change of the normalized magnetization intensity with time during the photomagnetization process of $\mathrm{Gd}_{25} \mathrm{Fe}_{75}$ alloy.

In the following calculation, the power density of the incident laser is $20 \mathrm{~mJ} / \mathrm{cm}^{2}$ and the injection current density is $0 \sim 10^{7} \mathrm{~A} / \mathrm{cm}^{2}$. When the injection current density does not reach the threshold value, as shown in Figure 5a,b, the magnetization of the two sublattices does not switch. When the injection current density exceeds the threshold value, as shown in Figure $5 c, d$, the higher the current density, and the faster the magnetization switching of Fe sublattice will occur. Fe and Gd sublattices will all transfer to a paramagnetic state due to the temperature rise up, but as a result of the atomic magnetic moment of Fe being smaller, the Fe sublattice will precede Gd melts to a paramagnetic state. The magnetization reversal of Gd sublattice will lag behind that of Fe sublattice, and the lag time is related to the magnitude of the injection current density. Calculation results are consistent with the work of Radu et al. [5]. The experimental results of Radu et al. are compared with our simulation results in Figure 6.

Figure 7 shows the time-resolved variation process of the normalized spin transfer torque in the case of reverse inversion of sublattice magnetization $\left(10^{6} \sim 10^{7} \mathrm{~A} / \mathrm{cm}^{2}\right)$. Where $\tau_{x}, \tau_{y}$ and $\tau_{z}$ are respectively the components of the normalized spin transfer torque in three directions in the Cartesian coordinate system; $T_{e}$ is the normalized electronic temperature; $J_{e}$ is the normalized current density. It can be found that the time for the current to reach the peak lags behind the time for the electron temperature to reach the peak, and when the current density gradually decays to 0 , the spin transfer torque effect also stops. In addition, the component of spin transfer torque in three directions has no obvious relationship with the injection current. This is because the torque is jointly affected by the current and the spin magnetic moments of the two sublattices. The constant precession of the spin moment will lead to constant change in the direction of the spin transfer torque, and then the spin transfer torque will react on the atomic spin. The magnitude of the current is the critical factor affecting the strength of this interaction. 
(a)

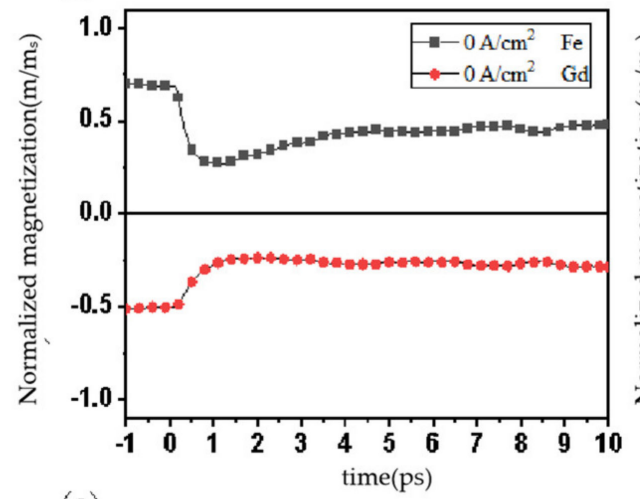

(c)

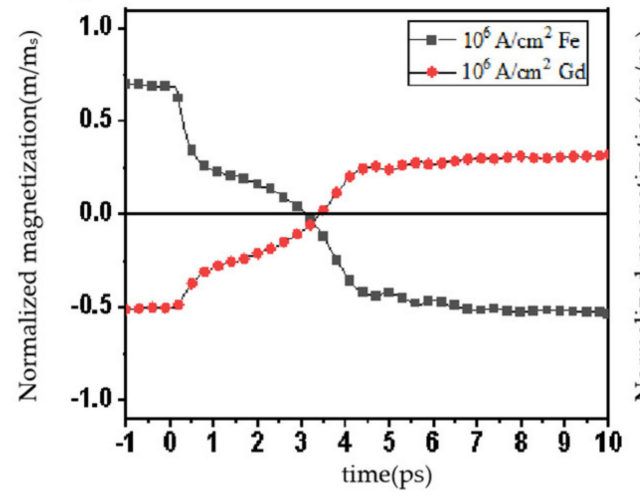

(b)

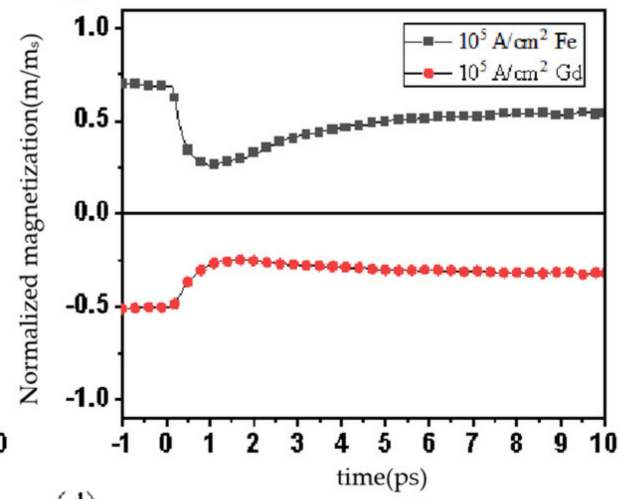

(d)

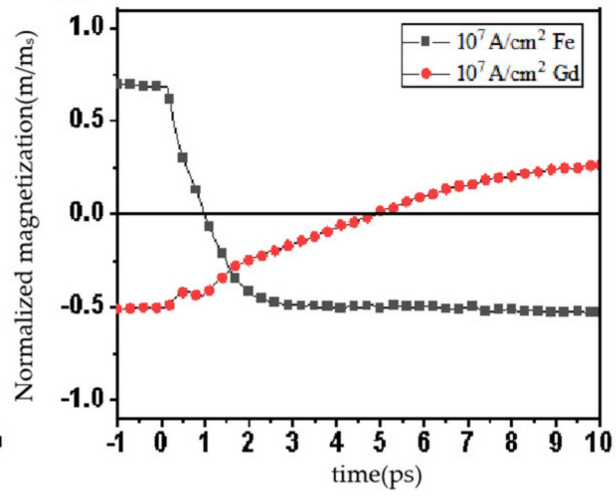

Figure 5. Time-resolved magnetization dynamics process of two sublattices at different current densities. For the situation $(\mathbf{a}, \mathbf{b})$, the magnetization of Fe and $\mathrm{Gd}$ is not reversed. The magnetization of two sublattices switching at the same time almost in (c). The switching time of Gd magnetization lags about several picoseconds behind $\mathrm{Fe}$ in (d). (a) The injection current density is $0 \mathrm{~A} / \mathrm{cm}^{2} ;(\mathbf{b})$ the injection current density is $10^{5} \mathrm{~A} / \mathrm{cm}^{2}$; (c) the injection current density is $10^{6} \mathrm{~A} / \mathrm{cm}^{2}$; (d) the injection current density is $10^{7} \mathrm{~A} / \mathrm{cm}^{2}$.

(a)

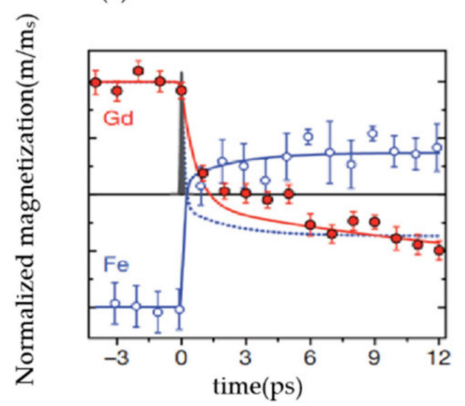

(b)

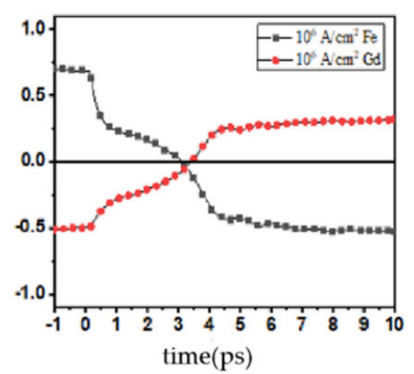

(c)

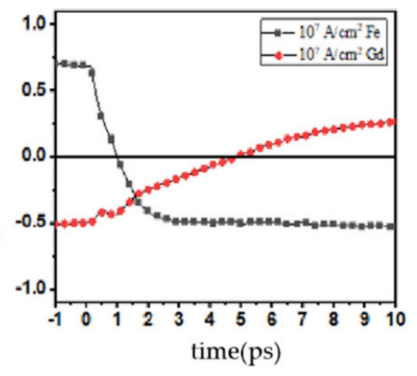

Figure 6. Comparison of normalized magnetization changes between experimental results and simulation results. (a) The experiment results performed by a single pulse of $60 \mathrm{fs}$ and a wavelength of $800 \mathrm{~nm}$ in $\mathrm{Gd}_{25} \mathrm{Fe}_{66.6} \mathrm{Co}_{9.4}$ alloy, and the laser power density is $4.4 \mathrm{~mJ} / \mathrm{cm}^{2}$; (b,c) the simulation results considering spin transfer torque effect. 


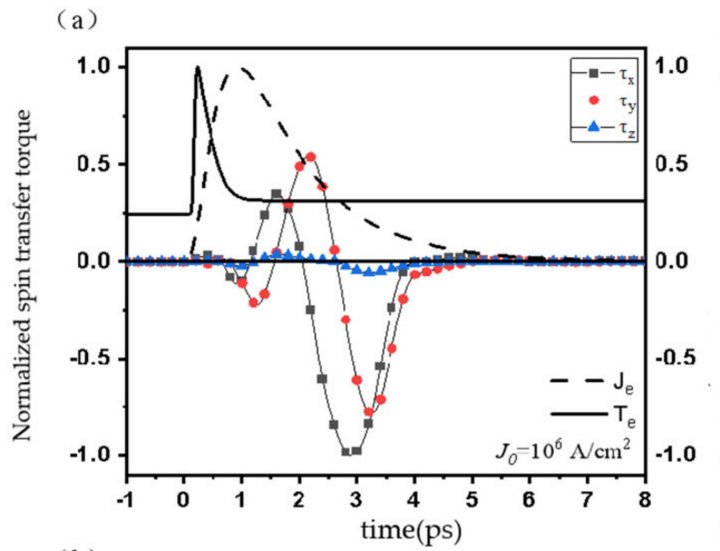

Figure 7. Time-resolved variation process of the normalized spin transfer torque in the case of sublattice magnetization flip (a) The injection current density is $10^{6} \mathrm{~A} / \mathrm{cm}^{2}$; (b) the injection current density is $10^{7} \mathrm{~A} / \mathrm{cm}^{2}$.

We put forward a new assumption to the AOS phenomenon occurring in GdFeCo amorphous alloy thin films: when the femtosecond laser single pulse acts on the recording medium, the electronic temperature rises rapidly, thermal perturbation makes the rare earth sublattice and transition metal sublattice demagnetization occurs quickly; however, the demagnetization rate of transition metal element sublattice is faster than that of rare earth element. Therefore, when Fe sublattice spins after complete demagnetization, Gd sublattice spins are still in a state of magnetization. The electronic temperature then drops rapidly below the Curie temperature, and the thermal disturbance does not play a critical role. At the same time, the superdiffusive spin current or the spin current caused by temperature gradient exceeds the threshold value, causing the spin transfer torque effect, which only works on the Fe sublattice. The effect of spin transfer torque causes the angular momentum change of Fe sublattice, and the magnetic moments of Fe atom procession occurs, and stochastic switching takes place on the magnetization of Fe. The magnetization of Fe sublattice reverses the magnetization of Gd sublattice due to exchange interaction. Finally, two sublattices maintain a stable antiferromagnetic coupling state, and the whole process of AOS is completed. This switching occurred on a subpicosecond time scale is nonlinear and it can be accomplished in a certain laser power density range. Due to it not involving the circular polarization of the laser pulses, the assumption is not only to adapt to describe the HI-AOS in RE-TM alloy materials, but is also to explain the HD-AOS in the same medium.

\section{Conclusions}

We suggested a new physical mechanism undertaking the AOS process in GdFeCo amorphous alloy thin film: the spin transfer torque induced by femtosecond laser single pulse acts on the spins of $\mathrm{Fe}$, leading to the reversal of Fe spins' orientation first; the elec- 
tronic temperature then quickly dropped below the Curie temperature, and the exchange interaction between Fe and Gd sublattices makes Gd spins switch. For this, we established the atomistic spin model to describe the magnetization dynamics of RE-TM materials. The results of the simulation show pretty good agreement with the experimental results carried out by Radu et al., showing that spin transfer torque takes an important role in the process: (1) the higher the power density of the incident single laser pulse, the lower the threshold of the spin current density required by AOS for RE-TM materials, and when the power density of the single laser pulse is within $10 \sim 20 \mathrm{~mJ} / \mathrm{cm}^{2}$, the order of the threshold between $10^{5} \sim 10^{7} \mathrm{~A} / \mathrm{cm}^{2}$ can be observed; (2) when the spin current density exceeds the threshold, the higher the current density is, and the faster the magnetization switching of Fe sublattice will be, the magnetization switching of Gd sublattice will lag behind that of Fe sublattice, and the lag time of Gd sublattices behind Fe relates to the magnitude of the spin current density.

The combination of ultra-fast laser-induced spin current and spin-mediated magnetization dynamics will give rise to many interesting applications. Understanding the underlying mechanism will be a great help for these applications, including improving the performance of magnetic storage technology. Our attempt for this conclusion is a bold try for the construction of a complete theoretical system of ultrafast laser-induced spin current magnetization dynamics.

Author Contributions: Methodology, H.H.; Software, H.H.; Validation, Z.Z. and R.W.; Formal Analysis, X.Z.; Resources, W.C.; Writing-Original Draft Preparation, H.H.; Supervision, H.W.; Funding Acquisition, C.X. All authors have read and agreed to the published version of the manuscript.

Funding: This work was funded by National Natural Science Foundation of China (NSFC) (61432007), the Creative Research Group Project of NSFC (61821003) and Science Technology Support Program of Hubei Province (2015BCE054).

Institutional Review Board Statement: Not applicable.

Informed Consent Statement: Not applicable.

Data Availability Statement: Not applicable.

Conflicts of Interest: The authors declare no conflict of interest.

\section{References}

1. Shokrollahi, H.J.J.O.M.; Materials, M. A review of the magnetic properties, synthesis methods and applications of maghemite. J. Magn. Magn. Mater. 2017, 426, 74-81. [CrossRef]

2. Stanciu, C.; Hansteen, F.; Kimel, A.V.; Kirilyuk, A.; Tsukamoto, A.; Itoh, A.; Rasing, T. All-optical magnetic recording with circularly polarized light. Phys. Rev. Lett. 2007, 99, 047601. [CrossRef]

3. Kimel, A.V.; Kirilyuk, A.; Rasing, T. Femtosecond opto-magnetism: Ultrafast laser manipulation of magnetic materials. Laser Photonics Rev. 2007, 1, 275-287. [CrossRef]

4. Stanciu, C.D.; Hansteen, F.; Kimel, A.; Tsukamoto, A.; Itoh, A.; Kirilyuk, A.; Rasing, T. Ultrafast interaction of the angular momentum of photons with spins in the metallic amorphous alloy GdFeCo. Phys. Rev. Lett. 2007, 98, 207401. [CrossRef] [PubMed]

5. Radu, I.; Vahaplar, K.; Stamm, C.; Kachel, T.; Pontius, N.; Dürr, H.A.; Ostler, T.; Barker, J.; Evans, R.; Chantrell, R.; et al. Transient ferromagnetic-like state mediating ultrafast reversal of antiferromagnetically coupled spins. Nat. Cell Biol. 2011, 472, 205-208. [CrossRef]

6. Savoini, M.; Medapalli, R.; Koene, B.; Khorsand, A.R.; Le Guyader, L.; Duò, L.; Finazzi, M.; Tsukamoto, A.; Itoh, A.; Nolting, F.; et al. Highly efficient all-optical switching of magnetization in GdFeCo microstructures by interference-enhanced absorption of light. Phys. Rev. B 2012, 86, 140404. [CrossRef]

7. Hohlfeld, J.; Gerrits, T.; Bilderbeek, M.; Rasing, T.; Awano, H.; Ohta, N.; Hohlfeld, J.; Gerrits, T.; Bilderbeek, M.; Rasing, T.; et al. Fast magnetization reversal of GdFeCo induced by femtosecond laser pulses. Phys. Rev. B 2001, 65, 012413. [CrossRef]

8. Zayats, A.V.; Smolyaninov, I.I.; Maradudin, A.A. Nano-optics of surface plasmon polaritons. Phys. Rep. 2005, 408, 131-314. [CrossRef]

9. Kano, H.; Mizuguchi, S.; Kawata, S. Excitation of surface-plasmon polaritons by a focused laser beam. J. Opt. Soc. Am. B 1998, 15, 1381-1386. [CrossRef] 
10. Stipe, B.C.; Strand, T.C.; Poon, C.C.; Balamane, H.; Boone, T.D.; Katine, J.A.; Li, J.-L.; Rawat, V.; Nemoto, H.; Hirotsune, A.; et al. Akemi Hirotsune, Magnetic recording at $1.5 \mathrm{~Pb} \mathrm{~m}-2$ using an integrated plasmonic antenna. Nat. Photonics 2010, 4, 484-488. [CrossRef]

11. Liu, T.M.; Wang, T.; Reid, A.H.; Savoini, M.; Wu, X.; Koene, B.; Granitzka, P.; Graves, C.E.; Higley, D.J.; Chen, Z.; et al. Nanoscale Confinement of All-Optical Magnetic Switching in TbFeCo-Competition with Nanoscale Heterogeneity. Nano Lett. 2015, 15, 6862-6868. [CrossRef]

12. Lambert, C.H.; Mangin, S.; Varaprasad, B.C.S.; Takahashi, Y.K.; Hehn, M.; Cinchetti, M.; Malinowski, G.; Hono, K.; Fainman, Y.; Aeschlimann, M.; et al. All-optical control of ferromagnetic thin films and nanostructures. Science 2014, 345, 1337-1340. [CrossRef] [PubMed]

13. Mentink, J.H.; Hellsvik, J.; Afanasiev, D.V.; Ivanov, B.A.; Kirilyuk, A.; Kimel, A.V.; Eriksson, O.; Katsnelson, M.I.; Rasing, T. Ultrafast spin dynamics in multisublattice magnets. Phys. Rev. Lett. 2012, 108, 057202. [CrossRef] [PubMed]

14. Ostler, T.; Barker, J.; Evans, R.; Chantrell, R.; Atxitia, U.; Chubykalo-Fesenko, O.; El Moussaoui, S.; Le Guyader, L.; Mengotti, E.; Heyderman, L.; et al. Ultrafast heating as a sufficient stimulus for magnetization reversal in a ferrimagnet. Nat. Commun. 2012, 3 , 666. [CrossRef]

15. Vahaplar, K.; Kalashnikova, A.M.; Kimel, A.V.; Gerlach, S.; Hinzke, D.; Nowak, U.; Chantrell, R.; Tsukamoto, A.; Itoh, A.; Kirilyuk, A.; et al. All-optical magnetization reversal by circularly polarized laser pulses: Experiment and multiscale modeling. Phys. Rev. B 2012, 85, 104402. [CrossRef]

16. Hohlfeld, J.; Stanciu, C.D.; Rebei, A. Athermal all-optical femtosecond magnetization reversal in GdFeCo. Appl. Phys. Lett. 2009, 94, 152504. [CrossRef]

17. Hopkins, P.E.; Ding, M.; Poon, J. Contributions of electron and phonon transport to the thermal conductivity of GdFeCo and TbFeCo amorphous rare-earth transition-metal alloys. J. Appl. Phys. 2012, 111, 103533. [CrossRef]

18. Pitaevskii, L. Electric forces in a transparent dispersive medium. Sov. Phys. JETP 1961, 12, 1008-1013.

19. Van Der Ziel, J.; Pershan, P.; Malmstrom, L. Optically-induced magnetization resulting from the inverse Faraday effect. Phys. Rev. Lett. 1965, 15, 190. [CrossRef]

20. Pershan, P.; Van Der Ziel, J.; Malmstrom, L. Theoretical discussion of the inverse Faraday effect, Raman scattering, and related phenomena. Phys. Rev. 1966, 143, 574. [CrossRef]

21. Li, Z.; Zhang, S. Magnetization dynamics with a spin-transfer torque. Phys. Rev. B 2003, 68, 024404. [CrossRef]

22. Li, Z.; Zhang, S. Thermally assisted magnetization reversal in the presence of a spin-transfer torque. Phys. Rev. B 2004, 69, 134416. [CrossRef]

23. Bertotti, G.; Serpico, C.; Mayergoyz, I.; Bonin, R.; d'Aquino, M. Current-induced magnetization dynamics in nanomagnets. J. Magn. Magn. Mater. 2007, 316, 285-290. [CrossRef]

24. Delgado, F.; Palacios, J.; Fernández-Rossier, J. Spin-transfer torque on a single magnetic adatom. Phys. Rev. Lett. 2010, 104, 02660. [CrossRef] [PubMed]

25. Graves, C.E.; Reid, A.H.; Wang, T.; Wu, B.; De Jong, S.; Vahaplar, K.; Radu, I.; Bernstein, D.P.; Messerschmidt, M.; Müller, L.; et al. Nanoscale spin reversal by non-local angular momentum transfer following ultrafast laser excitation in ferrimagnetic GdFeCo. Nat. Mater. 2013, 12, 293. [CrossRef] [PubMed]

26. Iacocca, E.; Liu, T.M.; Reid, A.H.; Fu, Z.; Dürr, H.A. Spin-current-mediated rapid magnon localisation and coalescence after ultrafast optical pumping of ferrimagnetic alloys. Nat. Commun. 2019, 10, 1756. [CrossRef] [PubMed]

27. Němec, P.; Rozkotová, E.; Tesarova, N.M.; Trojánek, F.; De Ranieri, E.; Olejnik, K.; Zemen, J.; Novák, V.; Cukr, M.; Malý, P.; et al. Experimental observation of the optical spin transfer torque. Nat. Phys. 2012, 8, 411-415. [CrossRef]

28. Freimuth, F.; Blügel, S.; Mokrousov, Y. Laser-induced torques in metallic ferromagnets. Phys. Rev. B 2016, 94, 144432. [CrossRef]

29. Schellekens, A.S.; Kuiper, K.K.; De Wit, R.R.; Koopmans, B.B. Ultrafast spin-transfer torque driven by femtosecond pulsed-laser excitation. Nat. Commun. 2014, 5, 4333. [CrossRef]

30. Choi, G.-M.; Min, B.-C.; Lee, K.-J.; Cahill, D.G. Spin current generated by thermally driven ultrafast demagnetization. Nat. Commun. 2014, 5, 4334. [CrossRef]

31. Mendil, J.; Cordones, P.N.; Chubykalo-Fesenko, O.; Walowski, J.; Santos, T.S.; Pisana, S.; Münzenberg, M. Resolving the role of femtosecond heated electrons in ultrafast spin dynamics. Sci. Rep. 2015, 4, 3980. [CrossRef] [PubMed]

32. Battiato, M.; Carva, K.; Oppeneer, P.M. Theory of laser-induced ultrafast superdiffusive spin transport in layered heterostructures. Phys. Rev. B 2012, 86, 024404. [CrossRef]

33. Seifert, T.; Jaiswal, S.; Martens, U.; Hannegan, J.; Braun, L.; Maldonado, P.; Freimuth, F.; Kronenberg, A.; Henrizi, J.; Radu, I.; et al. Efficient metallic spintronic emitters of ultrabroadband terahertz radiation. Nat. Photon 2016, 10, 483-488. [CrossRef]

34. D'Aquino, M. Nonlinear Magnetization Dynamics in Thin-Films and Nanoparticles. Available online: http://wpage.unina.it/ mdaquino/PhD_thesis/main/main.html (accessed on 30 June 2021).

35. Serpico, C.; Bertotti, G.; D'Aquino, M.; Bonin, R.; Mayergoyz, I. Transient dynamics leading to self-oscillations in nanomagnets driven by spin-polarized currents. IEEE Trans. Magn. 2005, 41, 3100-3102. [CrossRef]

36. Tserkovnyak, Y.; Brataas, A.; Bauer, G.E. Theory of current-driven magnetization dynamics in inhomogeneous ferromagnets. J. Magn. Magn. Mater. 2008, 320, 1282-1292. [CrossRef] 